\title{
Factores de riesgo asociados a diabetes por embarazo en pacientes atendidas en Centro de Salud Jipijapa
}

\section{Risk factors associated with diabetes due to pregnancy in patients treated at the Jipijapa Health Center}

\section{Factores de riesgo asociados a diabetes}

\author{
Karen Laura Macías Rodríguez ${ }^{(1)}$ \\ Johanna Mabel Sánchez Rodríguez ${ }^{(2)}$ \\ Jazmín Beatriz Anzules Guerra ${ }^{(3)}$ \\ Mirella Cedeño Holguín ${ }^{(4)}$
}

(1) Universidad Estatal del Sur de Manabí. e-mail: karma_21@live.com

(2) Universidad Laica Eloy Alfaro de Manabí. e-mail: dramabelsan24@ @otmail.com

(3) Universidad Técnica de Manabí. e-mail: mmmin1@ @otmail.com

(4) Universidad Estatal del Sur de Manabí. e-mail: doloresmirella@ hotmail.com

Contacto: karma_21@live.com

Recibido: 30-04-2020

Aprobado: 18-06-2020

\section{Resumen}

La diabetes es considerada una de las enfermedades metabólicas con mayor prevalencia en la población adulta a nivel mundial. El presente artículo tiene como objetivo, identificar los factores de riesgo asociados a la Diabetes por embarazo en las pacientes atendidas en el Centro de Salud Jipijapa, Ecuador. El tipo de estudio fue cuantitativo, descriptivo y analítico con respecto a los conocimientos, actitudes sobre los factores de riesgos asociados a la diabetes gestacional. La población fue 75 embarazadas entre las semanas 28 y 36 semanas de gestación. Se utilizó una encuesta para evaluar los conocimientos de las gestantes en lo relacionado a diabetes gestacional. Los principales resultados destacan que el $81 \%$ de la población encuestada no conocen que es la diabetes gestacional, el 95\% no realizaron asesoría preconcepcional, es decir son embarazos no planificados y no cuentan con un control donde se logre identificar las posibles complicaciones que se pueden presentar en el embrazo. Se logró identificar como principales factores de riesgo, la obesidad, la multiparidad, los antecedentes familiares relacionados con diabetes y la macrosomía en embarazos anteriores. Se concluyó que la falta de controles preconcepcionales y de conocimiento relacionados a diabetes gestacional, los factores de riesgo en las mujeres en estado de gestación representan una problemática de gran proporción y aumenta el riesgo de padecer diabetes gestacional, además la demora de atención al inicio del embarazo, controles durante el embarazo no periódicos y falta de ecografías oportunas no permiten un diagnóstico oportuno de diabetes gestacional.

Palabras claves: Diabetes gestacional, macrosomía, obesidad, antecedentes familiares.

\section{Sumary}

Diabetes is considered one of the metabolic diseases with the highest prevalence in the adult population worldwide. The objective of this article is to identify the risk factors associated with diabetes due to pregnancy in patients treated at the Jipijapa Health Center, Ecuador. The type of study was quantitative, descriptive and analytical regarding knowledge, attitudes about risk factors associated with gestational diabetes. The population was 75 pregnant women between 28 and 36 weeks of gestation. A survey was used to assess the knowledge of pregnant women regarding gestational diabetes. The main results highlight that $81 \%$ of the surveyed population do not know what gestational diabetes is, $95 \%$ did not perform preconception counseling, that is, they are unplanned pregnancies and do not have a control where it is possible to identify the possible complications that can be present in the embrace. Obesity, multiparity, family history related to diabetes and macrosomia in previous pregnancies were identified as the main risk factors. It was concluded that the lack of preconceptional controls 
and knowledge related to gestational diabetes, risk factors in women in pregnancy represent a large proportion of problems and increases the risk of suffering from gestational diabetes, in addition to the delay in care at the beginning of the Pregnancy, non-periodic controls during pregnancy and lack of timely ultrasounds do not allow a timely diagnosis of gestational diabetes.

Key words: Diabetes gestational, macrosomia, obesity, family history.

\section{Introducción}

La diabetes es considerada una de las enfermedades metabólicas con mayor prevalencia en la población adulta. Es una enfermedad crónica que aparece cuando el páncreas no produce insulina suficiente o cuando el organismo no utiliza eficazmente la insulina que produce Organización Mundial de Salud (OMS, 2017; OMS, 2018). Este tipo de enfermedad no controlada se considera hiperglucemia o aumento del azúcar en la sangre, que puede causar daños graves a muchos órganos y sistemas del cuerpo humano, mientras más se tarde un diagnóstico de diabetes mellitus más grave puede ser el daño causado por la enfermedad.

Según la OMS (2016), en su informe mundial sobre la diabetes, señala que esta enfermedad puede producir complicaciones en muchas partes del cuerpo y aumentar el riesgo general de morir prematuramente. Algunas de las complicaciones que se describen es lo concerniente al infarto del miocardio, los accidentes cerebrovasculares, la insuficiencia renal, la amputación de miembros inferiores, la perdida de agudeza visual y neuropatía. También afirman que en el embarazo, la diabetes mal controlada puede aumentar el riesgo de muerte fetal y otras complicaciones.

La Asociación Americana de Diabetes (ADA) señala que, "la diabetes gestacional se inicia cuando el cuerpo no puede producir ni utilizar toda la insulina que necesita para el embarazo. Sin suficiente insulina, la glucosa no puede salir de la sangre y convertirse en energía, esto se llama hiperglucemia" (ADA, 2015).Se puede dividir en dos grupos a las mujeres con diabetes en el embarazo: son aquellas cuya intolerancia a la glucosa se hace presente durante la gestación (diabetes gestacional) y las cuales en las que la diabetes antecede el embarazo (diabetes pre gestacional).

El Centro de Salud Jipijapa, asevera que la problemática existente en las mujeres gestantes atendidas en el centro de referencia, se centra en la falta de conocimiento y cultura en lo concerniente a los problemas de salud que puede causar la diabetes en embarazo, la falta de interés, la poca información recibida, la falta de atención preconcepcional y la demora al recibir atención al inicio del embarazo no permiten a los profesionales de salud la identificación oportuna y apropiada de los factores de riesgo y obtener de esta manera poder realizar diagnostico apropiado y oportuno.

El presente trabajo tiene como objetivo identificar los factores de riesgo asociados a la Diabetes por embarazo en las pacientes atendidas en el Centro de Salud Jipijapa, Ecuador.

\section{Diabetes Gestacional}

Según la OMS (2016), la diabetes que aparece por primera vez durante la gestación es considerada diabetes gestacional una de las enfermedades metabólicas que frecuentemente afecta el embarazo, poniendo en riesgo tanto a la madre como al producto. Se calcula que, a escala mundial, unas de cada siete mujeres embarazadas pueden padecer hiperglucemia, que en el $85 \%$ de los casos corresponden a diabetes gestacional (DG). La proporción de mujeres afectadas puede llegar al $30 \%$, pero según la literatura científica muchos casos de diabetes gestacional no se diagnostican, lo cual acarrea consecuencias potencialmente mortales para a madre y el bebé. También se ha notificado que en el embarazo representa también un gran índice de morbilidad y mortalidad en las mujeres que lo padecen que aumenta potencialmente según los factores de riesgo que rodean a la persona.

Por otra parte este tipo de patología mal controlada puede hacer daño tanto a la madre como al futuro bebe. Cuando este tipo de enfermedad se presenta, el páncreas funciona horas adicionales para producir insulina suficiente, pero esta no baja el nivel de glucosa en la sangre, lo que produce que la glucosa adicional en la sangre atraviese la placenta y con esto el futuro bebé tenga un alto nivel de glucosa y, a consecuencia el páncreas del producto produzca más insulina debido a que recibe más energía de la que necesita para crecer normalmente, almacenando como grasa la energía adicional, lo que conlleva a macrosomía o un bebe gordo, además de enfrentar problemas al nacer como hipoglicemia, problemas respiratorios, riesgo de obesidad y desarrollar diabetes tipo II de adultos (Herranz, 2014; Chancay, 2017; MayoClinic, 2020).

Otros estudios realizados por la OMS (2016), aseveran que la diabetes gestacional aumenta el riesgo de sufrir problemas obstétricos, como preclamsia, y también de tener cesárea y parto prematuro, debido probablemente a los bebes 
macrosomicos. En cuanto a los riesgos a largo plazo, la DG indica un riesgo importante de diabetes materna futura $y$ de enfermedades cardiovasculares. Para la descendencia, la prematuridad al nacer puede conducir a problemas pulmonares y a ictericia. El tamaño grande del bebe puede causar traumatismos en el parto, ocasionalmente con distocia de hombros, necesidades de atención neonatal por problemas respiratorios, hiperbilirrubinemia y glucemia baja.

En este sentido otros autores señalan que los bebes macrosómicos tienen más propensión al desarrollo futuro de intolerancia a la glucosa y del síndrome metabólico y, en algunas poblaciones a la diabetes. Es muy común que la diabetes gestacional desaparezca luego del parto, pero una vez que esta se ha presentado durante el embarazo existe la posibilidad de desarrollar nuevamente esta enfermedad en futuros embarazos o diabetes mellitus dentro de algunos años, con mucha más frecuencia si la obesidad se encuentra presente como factor de riesgo (Cueva, 2016; OMS 2017).

El éxito en el manejo de las embarazadas con diabetes se fundamenta en que los pacientes con diabetes pre-gestacional deben estar bien controladas desde el punto de vista metabólico, desde antes de la concepción y permanecer así durante la evolución del embarazo y con respecto a las diabéticas gestacionales, el peligro radica en desestimarlas y no diagnosticarlas a tiempo (Nappa,2015; Medina y Tomala 2018).

Según el Instituto Nacional de Estadísticas y Censos (INEC) la diabetes es la segunda causa de muerte, después de las enfermedades isquémicas del corazón, el número de fallecidos por diabetes en 10 años desde el 2007 hasta el 2016 aumentó en un 51\%, de las cuales en el 2016, 2628 eran mujeres (INEC, 2020). En estudios realizados se afirma que en Ecuador 6 de cada 10 personas no realizan ningún tipo de deporte, los tres alimentos con mayor consumo en los hogares son arroz blanco, el pan corriente de trigo y colas y gaseosas, lo que conlleva a una mala alimentación, además 6 de cada 10 ecuatorianos tienen sobrepeso, todos estos son factores que incrementan el riesgo de padecer diabetes.

En este sentido el índice de masa corporal (IMC) elevado, el sedentarismo, madres multíparas, la mala alimentación, las condiciones socioeconómicas inadecuadas, la falta de control en los embarazos, antecedentes personales y familiares, son los factores de riesgo más frecuentes y comunes para desarrollar diabetes por embarazo. En el sistema de notificación epidemiológica anual del MSP del Ecuador, la DG muestra un incremento sostenido en el periodo contenido entre 1994 y 2009, ascenso notablemente más pronunciado en los tres últimos años (Publica, 2014).

También se reporta que la tasa se incrementó de 142 por 100.000 a 1084 por 100.000 habitantes, con mayor prevalencia en mujeres de la costa ecuatoriana, y en especial de la provincia de Manabí. Este fenómeno se repite entre 2009 y 2013 (Publica, 2014). De acuerdo a los datos publicados por la Dirección Nacional de Vigilancia Epidemiológica en el 2017 se reportaron 106.008 casos de diabetes mellitus en Ecuador de los cuales 19.489 casos se encuentran en la provincia de Manabí, lo cual la ubica en una de las provincias con mayores casos de diabetes mellitus en la región costa.

Este centro de salud se encuentra ubicado en la zona urbana de la ciudad, es en esta institución donde se realizan los controles periódicos durante la gestación debido a pertenecer al primer nivel de salud del Ministerio de Salud Pública. Cuenta con un club de embarazadas donde se brinda consejería y charlas constantes relacionadas a controles y posibles complicaciones relacionadas con el estado de las pacientes, la atención a las mujeres gestantes son atendidas en el área de ginecología que cuenta con tres consultorios que atienden aproximadamente a 70 pacientes diariamente, el Centro de Salud cuenta con 1 Ginecólogo quien también realiza las ecografías ginecológicas y con 2 Obstetras, los turnos son repartidos por el Call Center del Ministerio de Salud Pública para dos de los consultorios y el restante atiende según la demanda del centro de salud (Ministerio de Salud Pública, 2020).

\section{Materiales y métodos}

Esta investigación se llevó a cabo por medio de un estudio descriptivo, prospectivo, cuantitativo $\mathrm{y}$ analítico, basado en los métodos inductivo y analítico, el universo estaba establecido por las 75 embarazadas entre las semanas 28 y 36 semanas de gestación multigestas que acuden al Centro de Salud Jipijapa, por controles gestacionales actualmente, debido a la muestra reducida no se realizó la técnica muestral.

La investigación se desarrolló en el Centro de Salud Jipijapa, Ecuador. La investigación se emprendió con pacientes embarazadas entre las 28 y 36 semanas de gestación, multíparas, se excluye a las primigestas por ser la multiparidad un factor de riesgo considerado para desarrollar diabetes gestacional.

Se aplicó la encuesta sobre conocimientos, actitudes y prácticas relacionadas a factores de 
riesgo con diabetes gestacional. Para la recolección de datos se procedió de la siguiente manera, se revisó la cantidad de embarazadas seleccionadas para la participación de la investigación que cumplieron con los criterios de inclusión, previa la autorización de la embarazada se procedió a realizar la encuesta, llenando los datos que aportaba la mujer gestante en relación a conocimiento de diabetes gestacional y a la identificación de los factores de riego que podían llevar a la mujer a desarrollar diabetes gestacional, además de identificar si fueron aplicadas las medidas establecidas en la Guía práctica clínica del Ministerio de Salud Púbica del Ecuador con la finalidad de prevenir el desarrollo de diabetes gestacional.

Las 75 embarazadas que formaron parte del estudio, cumplieron con los parámetros que ameritaba el instrumento posteriormente los datos fueron ingresados y tabulados en la base de datos de Microsoft Excel, para su análisis estadístico se realizó con un intervalo de confianza del 95\%,

\begin{tabular}{|l|l|l|}
\hline $\begin{array}{l}\text { Conocimiento de diabetes } \\
\text { gestacional }\end{array}$ & N. de respuestas & $\%$ \\
\hline $\mathrm{Si}$ & 11 & 18.33 \\
\hline No & 49 & 81.67 \\
\hline Total & 60 & 100 \\
\hline
\end{tabular}

Tabla 1. Conocimiento de diabetes gestacional

En la tabla 2 se identifican los principales factores de riesgos relacionados con diabetes gestacional encontrados en el estudio y su incidencia en las gestantes atendidas en el Centro de Salud Jipijapa, por medio de esta tabla se identifica a la multiparidad como un factor de riesgo presente en el $100 \%$ de las encuestadas, seguida de los antecedentes familiares de diabetes con un $60 \%$, el sobrepeso con $57 \%$, los antecedentes personales de diabetes gestacional con $20 \%$ y la macrosomía en un $15 \%$. La multiparidad y los antecedentes familiares de diabetes son considerados factores de riesgo para desarrollar diabetes en el embarazo, siendo estos indicadores que padecen la mayor parte de las embarazadas encuestadas, todo tipo de factores de riesgo es necesario identificarlos antes del embarazo o al inicio del mismo con la finalidad de tomar las acciones necesarias para conservar la integridad del estado de salud de la paciente.

En la tabla 2 se identifican los principales factores de riesgos relacionados con diabetes gestacional encontrados en el estudio y su incidencia en las gestantes atendidas en el Centro de Salud Jipijapa, por medio de esta tabla se identifica a la multiparidad como un factor de riesgo presente en el $100 \%$ de las encuestadas, seguida de los

\begin{tabular}{|l|l|l|}
\hline Factores de riesgo & f & $\%$ \\
\hline Multiparidad & 60 & 100 \\
\hline
\end{tabular}

Dentro de los aspectos bioéticos se garantizó la confidencialidad de las participantes en la que se aseguró el anonimato y reserva de datos en cada una de las encuestas realizadas.

\section{Resultados}

Los resultados reflejan que en la pregunta relacionada con el conocimiento de diabetes gestacional, el $81.67 \%$ indicaron que no conocen, mientras que el $18.33 \%$ si conocen, lo que evidencia que la mayor parte de las encuestadas desconocen lo relacionado a diabetes gestacional, lo que no permite un adecuado autocuidado, así como la ausencia de identificación a tiempo de los factores de riesgo propios que pueden presentarse antes y durante el embarazo y acudir al médico en tiempo adecuado, evitando posibles complicaciones en su salud, se aprecia en la tabla 1 .

antecedentes familiares de diabetes con un $60 \%$, el sobrepeso con $57 \%$, los antecedentes personales de diabetes gestacional con $20 \%$ y la macrosomía en un $15 \%$. La multiparidad y los antecedentes familiares de diabetes son considerados factores de riesgo para desarrollar diabetes en el embarazo, siendo estos indicadores que padecen la mayor parte de las embarazadas encuestadas, todo tipo de factores de riesgo es necesario identificarlos antes del embarazo o al inicio del mismo con la finalidad de tomar las acciones necesarias para conservar la integridad del estado de salud de la paciente. 


\begin{tabular}{|l|l|l|}
\hline Antecedentes familiares de diabetes & 37 & 66 \\
\hline Sobrepeso. & 34 & 57 \\
\hline $\begin{array}{l}\text { Guanacia de peso inadecuada en el embarazo } \\
\text { actual }\end{array}$ & 22 & 37 \\
\hline $\begin{array}{l}\text { Antecedentes personales de diabetes } \\
\text { gestacional. }\end{array}$ & 12 & 20 \\
\hline Macrosomía en embarazos anteriores & 9 & 15 \\
\hline
\end{tabular}

Tabla 2. Incidencia de factores de riesgo identificado en mujeres gestantes atendidas en Centro de Salud Jipijapa

\section{Discusión}

Dentro de los criterios de la atención médica a las embarazadas según el modelo de atención integral en salud MAIS (Publica, 2012), se toman en cuenta muchos aspectos importantes que como hemos podido evidenciar en el presente estudio no se logran completar en el proceso de embarazo, se debe implementar estrategias para fomentar e incentivar a las madres que desean embarazarse a realizar consultas pregestacionales, con la finalidad de disminuir los factores de riesgo encontrados, llevar y desarrollar un embarazo saludable y por supuesto preparar el cuerpo para el embarazo antes de desarrollarlo.

Se concuerda con otras investigaciones que aseveran que el éxito para enfrentar esta enfermedad, se fundamenta en el control, desde el punto de vista metabólico, desde antes de la concepción y al continuar con éste durante la evolución del embarazo y, respecto a las pacientes diabéticas gestacionales, en el diagnóstico temprano. En estas pacientes deben considerarse los factores de riesgo de diabetes gestacional (Medina, 2015; Lozano, Betancourth, y Melendez, 2016; Medina et al. 2017).

Se requiere además la aplicación de la guía práctica clínica diagnóstico y desarrollo de la diabetes en el embarazo (pregestacional y gestacional), desde la preconcepción hasta el final del embarazo, intentando en lo posible que los exámenes y ecografías se las realice en el tiempo establecidos, por lo que se concuerda por lo planteado por otros autores que hacen referencia a la guía (Publica, 2014; Ledesma,2017; Peremo y Ortega 2018).

Es fundamental hacer referencia a la realización de glicemia capilar en el primer contacto con la embarazada y trabajar conjuntamente en vinculación con la sociedad, el personal de salud y la comunidad universitaria inmiscuida en el área de la salud deben trabajar en la difusión de información hacia la sociedad.

\section{Conclusiones}

El desconocimiento de la enfermedad y la percepción errónea del verdadero estado nutricional de las gestantes dificulto la identificación de los factores de riesgo que inciden en el desarrollo de la patología en las usuarias quienes dejando a un lado la atención médica oportuna, solo recurrían a esta ante el desarrollo síntomas que inducían un riesgo para la salud de la madre y del producto, aumentando la morbilidad y mortalidad materna.

Dentro de los factores de riesgo asociados a diabetes gestacional identificados en el estudio constaron la multiparidad, factor que comparten todas las gestantes, seguido de los antecedentes familiares de diabetes, además de obesidad, antecedentes personales de diabetes gestacional y macrosomía en los productos de embarazos anteriores, lo que convierte a esta patología en un importante problema de salud pública.

En su mayoría provienen de las zonas rurales, es posible que se deba al bajo nivel de instrucción que presentan o a la falta de conocimiento con respecto al embarazo, se evidencia además un nivel socioeconómico bajo.

\section{Bibliografías}

ADA. (2015). Diabetes gestacional. American Diabetes Association. Recuperado de http://www.diabetes.org/es/informacionbasica-de-la-diabetes/diabetesgestacional/que-es-la-diabetesgestacional.html

Benzadón, M., Forti, L., y Sinay, I. (2014). Actualización en el diagnóstico de la diabetes . Scielo, 74(1). Rev. Cubana Pediatr.88, (3).Recuperado de http://scielo.sld.cu/pdf/ped/v88n3/ped0703 316.pdf

Chancay, C. (2017). Diabetes gestacional y embarazo. Recuperado ede https://www.cdc.gov/pregnancy/spanish/di abetes-gestational.html

Cueva, J. (2016). Autocuidados en el embarazo. Recuperado 
https://www.ecuadorencifras.gob.ec/diabet es-segunda-causa-de-muerte-despues-delas-enfermedades-isquemicas-del-corazon/

Herranz, L. (2014). Tipos de diabetes. Recuperado de

http://www.fundaciondiabetes.org/infantil/ 177/tipos-de-diabetes-ninos

INEC. (2020). Diabetes, segunda causa de muerte después de las enfermedades isquémicas

Ledesma, A. (2017). Pruebas y diagnóstico para la diabetes gestacional. Recuperado de https://www.niddk.nih.gov/healthinformation/informacion-de-lasalud/diabetes/informacion-general/quees/gestacional/pruebas-diagnostico

Lozano , A., Betancourth, W., \& Melendez, J. (2016). El sobrepeso y obesidad en el embarazo: Complicaciones y Manejo. Recuperado de http://www.archivosdemedicina.com/medi cina-de-familia/sobrepeso-y-obesidad-enel-embarazo-complicaciones-ymanejo.php?aid=11135

Mayo-Clinic.(2020). Diabetes Gestacional. Recuperado de https://www.mayoclinic.org/eses/diseases-conditions/gestationaldiabetes/symptoms-causes/syc-20355339

Medina, E., Sánchez, A., Hernández, A., Martínez, M., Jiménez, C., Serrano, I., Maqueda, A., Islas, D., y Cruz, M. (2017). Diabetes gestacional. Diagnóstico y tratamiento en el primer nivel de atención. Medicina interna de México, 33(1), 91-98. Recuperado en de http://www.scielo.org.mx/scielo.php?script =sci_arttext\&pid=S0186$\underline{48662017000100091 \& \operatorname{lng}=\mathrm{es} \& \operatorname{tlng}=\mathrm{es}}$.

Medina, F., y Tomala, T. (2012). Factores Asociados a la diabetes gestacional en pacientes de 25 a 40 años en un Hospital de Especialidad de la Ciudad de Guayaquil. Tesis de Lic. en enfermería. Recuperado de http://repositorio.ug.edu.ec:

http://repositorio.ug.edu.ec/bitstream/redug /1842/1/FACTORES\%20DE\%20RIESGO S\%20MATERNOS\%20Y\%20FETALES \%20EN\%20MADRES\%20QUE\%20PAD ECEN\%20DIABETES\%20GESTACION AL.pdf

Medina, V. (2015). Cómo controlar el peso durante el embarazo. Recuperado de https://www.guiainfantil.com/blog/305/co mo-controlar-el-peso-durante-elembarazo.html

Ministerio de Salud Pública, Ecuador. (2020). prevención y autocuidado son claves para controlar la diabetes. Recuperado de https://www.salud.gob.ec/ministerio-desalud-prevencion-y-autocuidado-sonclaves-para-controlar-la-diabetes/

Nappa, K. (2015). El tratamiento de la diabetes gestacional. Recuperado de http://www.diabetes.org/es/informacionbasica-de-la-diabetes/diabetesgestacional/el-tratamiento-de-ladiabetes.html

OMS. (2016). Informe mundial sobre diabetes . Organizacion Mundial de la Salud.Recuperado de https://www.who.int/diabetes/globalreport/es/

OMS. (2017). Diabetes. Recuperado de http://www.who.int/es/news-room/factsheets/detail/diabetes

OMS. (2018). Es hora de actuar: Informe de la Comisión Independiente de alto nivel de la OMS sobre Enfermedades no Transmisibles. Informe, Organizacion Mundial de la Salud, Ginebra.

Peremo, B., \& Ortega, S. (2018). Consulta preconcepcional Diabetes: Recuperado de https://www.webconsultas.com/embarazo/ quedarse-embarazada/que-es-una-consultapreconcepcional

Publica, M. D. (2014). Diagnóstico y tratamiento de la diabetes en el embarazo (pregestacional y gestacional). Guia de Práctica Clínica. Ministerio de Salud Pública, Quito. 